\title{
Lesiones cutáneas en educadores físicos costarricenses
}

José Moncada- Jiménez ${ }^{1,2}$, Maureen Meneses-Montero ${ }^{1}$, Benjamín Hidalgo-Matlock ${ }^{3}$, Caridad Granados-C havarría ${ }^{3}$

\author{
Resumen
}

1. Escuela de Educación Física y Deportes, U niversidad de Costa Rica

2. Escuela de Medicina, Universidad de Costa Rica

3. Servicio de Dermatología,H ospital Rafael Ángel Calderón Guardia,C aja Costarricense de Seguro Social

\begin{abstract}
Abreviaturas: CIE,C omission Internationale de L'Eclairage ; FPU, factor de protección a la radiación ultravioleta; RUV, radiación ultravioleta;SPSS,Statistical package for the social sciences; UVA, radiación ultravioleta $A$;UVA I,radiación ultravioleta I;UVA II radiación ultravioleta II;UVB,radiación ultravio leta B UVC, radiación ultravioleta C
\end{abstract}

Correspondencia: M.Sc. José Moncada Jiménez.A pdo. 239-1200 (Pavas), San José, C osta Rica. Fax. (506) 225-0749. E-mail: jmoncada@ cariari.ucr.ac.cr

ISSN 0001-6002/2004/46/3/132-138 Acta Médica Costarricense,(@2004 Colegio de Médicos y Cirujanos

\begin{abstract}
Justificación y objetivo: Los educadores físicos representan una población laboral que se expone crónicamente a la radiación solar. No se han descrito las lesiones cutáneas en este grupo de personas. El propósito del estudio fue describir la incidencia de lesiones cutáneas en educadores físicos costarricenses.

Métodos: En total, participaron voluntariamente 23 hombres (48.9\%) y 24 mujeres (51.1\%), a quienes se les aplicó un cuestionario. Los 47 participantes fueron revisados por un médico del Servicio de Dermatología del Hospital R. A. Calderón Guardia de la Caja Costarricense de Seguro Social. Se obtuvo información demográfica, fenotipo, salud general y horario de trabajo, prendas de vestir utilizadas para laborar, protección y cuidado de la piel, historia familiar y personal de cáncer de piel, y ubicación anatómica de las lesiones.

Resultados y discusión: Las lesiones cutáneas se ubicaron en las regiones del rostro, el pecho, la espalda alta, los brazos y los muslos. Hubo 3 casos con antecedentes familiares de cáncer de piel, y una persona presentó antecedentes personales (no melanoma). Se encontraron lesiones premalignas en 5 sujetos $(6.3 \%)$. Se recomienda evitar la exposición solar; una revisión anual con el dermatólogo; un autoexamen de piel completo mensualmente; el uso de prendas de vestir cuya translucidez sea poca, hechas de un material como el algodón, idealmente de color claro; también el uso de gorra o sombrero, anteojos oscuros, filtros solares, y si fuera posible, cambiar horarios de trabajo para horas cuando ocurre la menor radiación solar.
\end{abstract}

Descriptores: dermatología, educadores, cáncer de piel

Recibido: 30 de septiembre de 2003

Aceptado: 27 de abril de 2004

Se ha indicado que el pigmento natural de la piel no puede brindar una protección completa contra la radiación ultravioleta (RUV) de la luz solar; en consecuencia, podrían generarse lesiones cutáneas que eventualmente se podrían convertir en algún tipo de cáncer ${ }^{1}$. En la literatura se han descrito diversos factores de riesgo para el cáncer de piel, entre los cuales se pueden citar el fototipo de piel, la raza, el sexo, la exposición al sol (aguda o crónica), la historia familiar, la edad (el adulto mayor es más propenso) y el estado inmunitario (las personas inmunosuprimidas son más susceptibles) ${ }^{1,2}$. Otros factores incluyen a quienes viven en altitudes, en poblaciones de zonas donde se reciben muchos días con sol al año, personas que trabajan al aire libre, e individuos que han sufrido tres o más quemaduras solares graves.

También se ha propuesto que las personas de piel blanca, de cabello rubio o rojo, y de ojos color verde o azul, tienen una mayor probabilidad de desarrollar cáncer del tipo no melanoma ${ }^{1,3,4}$. Estas características fenotípicas son comunes en las poblaciones de tipo anglosajona o de origen mediterráneo, a las que además se les dificulta obtener un bronceado y que suelen quemarse luego de la exposición al sol (se tornan de color rojo). 
En los países centroamericanos no existe una población mayoritaria con características fenotípicas como las mencionadas (piel blanca, cabello rubio, ojos color verde o azul). A pesar de lo anterior, las estadísticas demuestran que en Costa Rica el cáncer de piel ocupó el primer lugar de incidencia entre las mujeres, y el segundo entre los hombres, en el trienio de 1994 a $1996^{5}$. Según datos del Registro Nacional de Tumores del Ministerio de Salud de Costa Rica, el 65\% de los tumores de piel se presentaron en mujeres y hombres de 65 años y más ${ }^{5}$.

De los tres tipos de cáncer de piel causados por la exposición crónica y excesiva al sol (epitelioma basocelular, carcinoma epidermoide y melanoma maligno), el epitelioma basocelular es el más común (75\% de todos los casos); sin embargo, los melanomas parecen ser los más agresivos y potencialmente mortales, en especial en las mujeres jóvenes ${ }^{~}$. En EEUU, el cáncer de piel es el más frecuentemente diagnosticado, siendo el melanoma el responsable de la mayor cantidad de muertes ${ }^{6}$.

Por la naturaleza de su oficio, una cantidad importante de educadores físicos se exponen diaria y crónicamente a la RUV del sol ${ }^{7}$, la cual podría relacionarse a la de los deportistas profesionales. A pesar del reconocido valor de la exposición solar para la salud, no se han llevado a cabo estudios descriptivos que permitan determinar si los educadores físicos presentan lesiones en la piel que pudiesen ser atribuibles a la exposición crónica y prolongada al sol. Por lo tanto, el propósito de la investigación fue describir las lesiones cutáneas de un grupo de educadores físicos, y proponer posibles relaciones para la aparición de cáncer de piel.

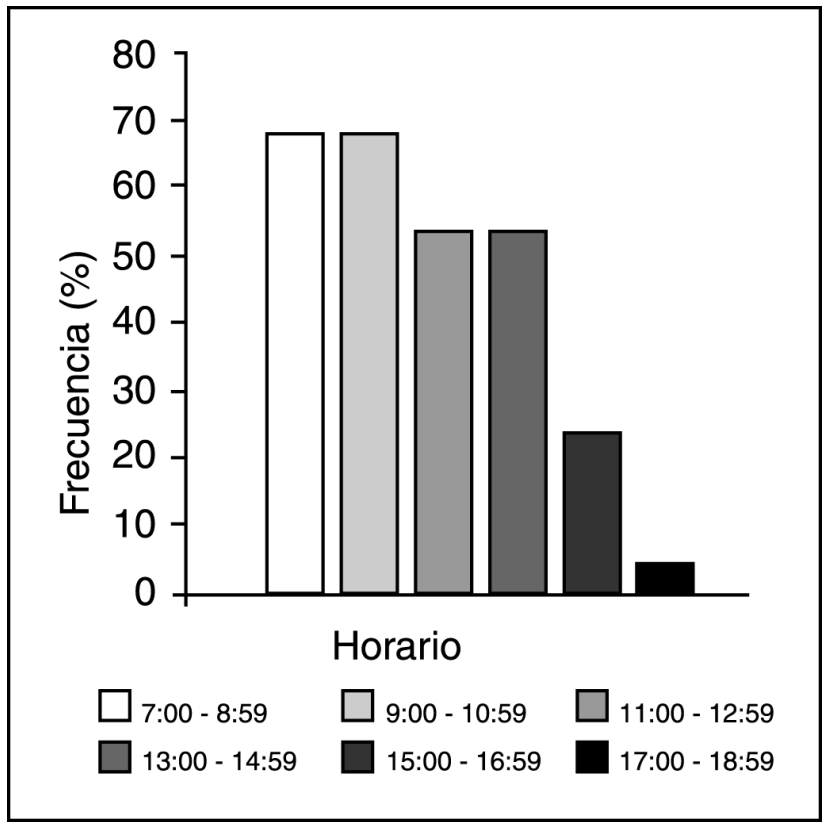

Figura 2. Frecuencia de exposición solar crónica, según horarios de trabajo, Costa Rica, 2003.
Materiales y métodos

Sujetos: El muestreo fue intencional y participaron educadores físicos provenientes de seis provincias de Costa Rica, quienes asistieron al IV Congreso Nacional de Educación Física realizado en marzo de 2003. Los participantes respondieron voluntariamente un cuestionario y se sometieron a una revisión por parte de un médico dermatólogo del Servicio de Dermatología del Hospital Rafael Ángel Calderón Guardia, de la Caja Costarricense de Seguro Social. En total, fueron revisados por el médico 47 educadores, 23 hombres $(48.9 \%)$ y 24 mujeres $(51.1 \%)$, quienes dieron su consentimiento para participar en el estudio.

Instrumentos: Se elaboró un cuestionario para obtener información demográfica, determinar el tipo de piel, datos relacionados con su cuidado y características del trabajo que realizan habitualmente los educadores físicos. Se efectúo un examen cutáneo completo y se aplicaron criterios clínicos de análisis de las lesiones pigmentadas sospechosas de cáncer de piel no melanoma y melanoma. A las lesiones pigmentadas se les realizó un análisis dermatoscópico, con un dermatoscopio de luz polarizada (Dermlite DL100 de 3GEN Corp., Dana Point, CA, USA), y se les aplicó la regla del ABCDE para la dermatoscopía, para su clasificación en benignas, dudosas y malignas; esta escala contempla criterios que consideran, un puntaje de 1.0 a 4.75 como lesiones benignas, de 4.75 a 5.45 como sospechosas por melanoma y mayores a 5.45 , como altamente sospechosas de melanoma.

Procedimientos: Los participantes se acercaron voluntariamente a un área acondicionada para que llenaran el cuestionario y fueran revisados por el médico dermatólogo. Cada uno llenó el cuestionario y luego fue atendido por el médico en un consultorio. El médico revisó minuciosamente a cada participante y anotó las lesiones observadas; estas fueron codificadas de acuerdo con un diagrama diseñado para tal fin (Figuras 3 y 4).

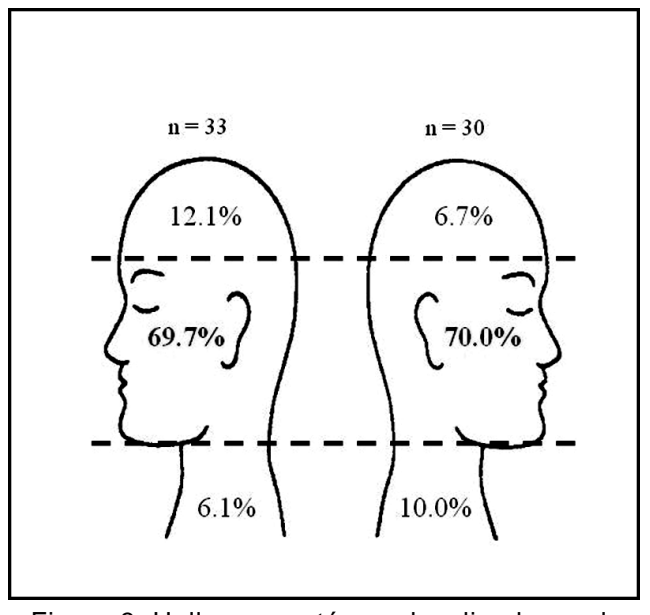

Figura 3. Hallazgos cutáneos localizados en la cabeza, Costa Rica, 2003. 


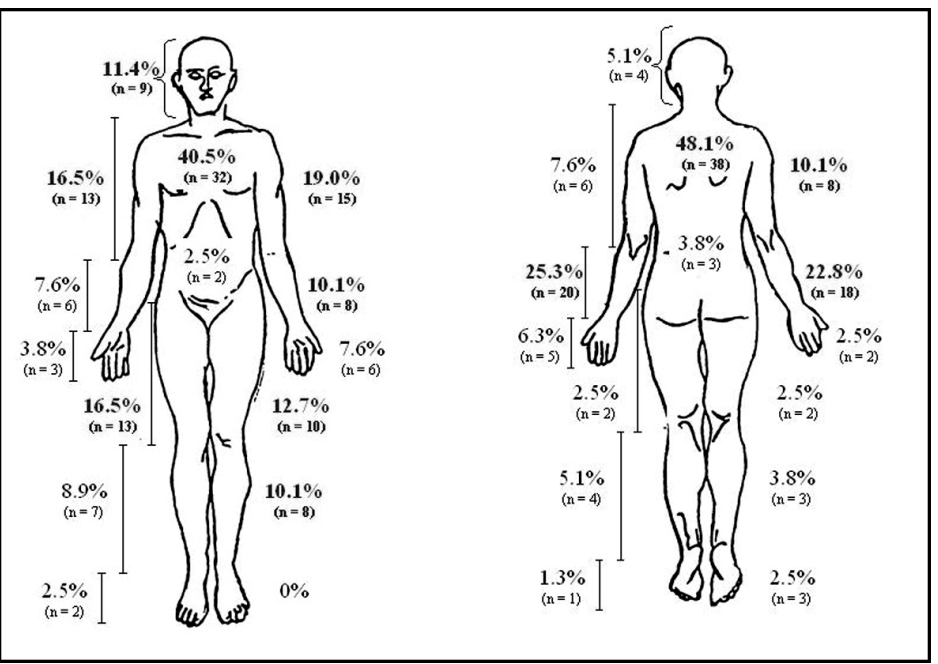

Figura 4. Hallazgos cutáneos en regiones anterior y posterior, Costa Rica, 2003.

Análisis estadísticos: Los cuestionarios fueron codificados y analizados con el SPSS, versión 8.0. Se obtuvieron estadísticas descriptivas para los datos continuos (media aritmética y desviación estándar) y porcentajes (para las variables categóricas).

\section{Resultados}

En el estudio hubo representación de 6 provincias de Costa Rica. La mayoría de los 47 educadores físicos que respondieron la encuesta provenían de San José (42.6\%) y Heredia $(31.8 \%)$. Hubo una pequeña cantidad de participantes de Alajuela $(8.5 \%)$, Cartago (6.4\%), Puntarenas (6.4\%) y Guanacaste $(4.3 \%)$. Se observó una población heterogénea en cuanto a edad, años de laborar como educadores físicos, años laborados al aire libre y número de horas aproximadas de trabajo al aire libre (Cuadro 1).

Los fototipos cutáneos se establecieron de I a VI y se relacionan con la tendencia de un individuo a quemarse o broncearse, luego de una hora de exposición solar a medio día ${ }^{7}$. Así, el fototipo de piel, según la clasificación de Fitzpatrick ${ }^{8}$, fue evaluado por el dermatólogo (Figura 1). En breve, la piel tipo I es aquella que siempre se quema y que nunca se broncea; la tipo II, aquella que siempre se quema y que algunas veces se broncea; la tipo III, aquella que se quema algunas veces y que siempre se broncea; tipo IV, es aquella que nunca se quema y que siempre se broncea; tipo V, es aquella moderadamente pigmentada, y la tipo VI, la piel negra.

De los 47 participantes, solamente una persona (2.1\%) declaró tener cabello color rubio natural, mientras que los demás señalaron tener cabello color negro u oscuro (49\%) y cabello color castaño o café

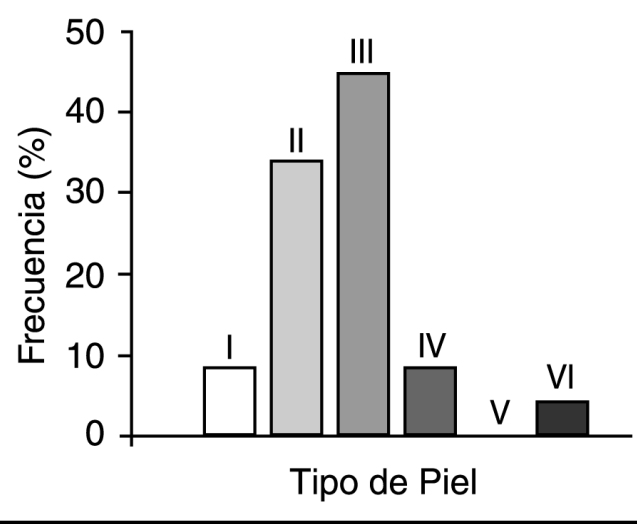

Figura 1. Tipos de piel de los 47 participantes evaluados, de acuerdo con la clasificación Fitzpatrick, Costa Rica, 2003.

(48.9\%). Por otra parte, el color de ojos café y miel fue el más reportado $(59.5 \%)$, mientras que los colores negro u oscuro (23.4\%) y verdes y azules (17\%) fueron reportados por una menor cantidad de participantes.

Al momento de la encuesta, el $23.4 \%$ reportó poseer alguna enfermedad, entre las cuales se mencionan: enfermedad de Graves, migraña, hipertensión arterial, gastritis, niveles elevados de triglicéridos, obesidad, sinusitis, asma, rinitis y alergias.

Se preguntó a cada participante el horario en el que regularmente laboraba, con el propósito de determinar el número aproximado de horas de exposición solar. En la Figura 2 se observa que más del 50\% de los educadores labora de 7:00 a.m. a 3:00 p.m.; es decir, se incluyen las horas cuando ocurre la mayor RUV.

Los educadores utilizan generalmente pantalón corto o pantaloneta $(72.3 \%)$ y camiseta con mangas cortas $(89.4 \%)$ para impartir las clases. Sin embargo, también visten pantalón deportivo (17\%) y camisetas sin mangas (14.9\%). Entre los accesorios más usados se encuentran la gorra o sombrero $(55.3 \%)$ y los anteojos (40.4\%), aunque no se sabe si utilizan

\section{Cuadro 1: Características de los 47 participantes evalua- dos en el IV Congreso Nacional de Educación Física. Costa Rica, 2003.}

\begin{tabular}{|lcccc|} 
Variable & M & \pm DS & Mín & Máx \\
\hline Edad (años) & 35 & 9 & 20 & 53 \\
Años de laborar como educador físico & 13.3 & 7.6 & 2 & 28 \\
Años laborados al aire libre & 7.9 & 6.9 & 0 & 28 \\
Horas diarias laboradas al aire libre & 5.0 & 2.5 & 1 & 10 \\
\hline
\end{tabular}

Nota: $\mathrm{M}=$ media aritmética; $\mathrm{DS}$ = desviación estándar; Mín = valor mínimo; Máx = valor máximo. 
anteojos de prescripción médica u oscuros. Una persona $(2.1 \%)$ aseveró que acostumbra utilizar camiseta con mangas largas para impartir lecciones.

El material más común en las prendas de vestir fue el tejido de algodón (77.8\%), también reportado como "tela de punto". Entre los encuestados $(\mathrm{n}=4)$, el tejido sintético $(11.2 \%)$ también fue utilizado regularmente. Los materiales sintéticos más comunes fueron la lycra®, el poliéster y el hilo. Otros incluyeron la seda $(2.8 \%)$, la lana $(2.8 \%)$ y poliéster/algodón (5.6\%).

La mayoría de educadores respondieron que generalmente utilizan una combinación de colores claros y oscuros en sus prendas $(57.8 \%)$, en oposición a aquellos que solo visten ropa con colores claros $(40 \%)$, o la minoría que viste únicamente colores oscuros $(2.2 \%)$.

En el cuestionario se formularon dos preguntas respecto al uso de lociones bronceadora y cremas con filtro solar. Solo 10 personas $(21.3 \%)$ afirmaron utilizar lociones bronceadoras. Por otra parte, respecto a los filtros solares, 27 personas $(57.4 \%)$ afirmaron utilizarlos. Adicionalmente, cuando se les consultó por el factor de protección solar de los productos (FPU), un $19.1 \%$ de los educadores manifestó usar un FPU $\leq$ 30 , mientras que el $21.3 \%$ indicó un FPU $\geq 35$. El 82.1\% de las personas que emplean el filtro solar, se lo aplican una o dos veces por día, mientras que un $14.3 \%$ indicó aplicárselo entre 3 y 4 veces diarias. El 3.6\% de los educadores señaló utilizar el filtro entre 5 y 6 veces por día.

Cuando se les preguntó cuánto tiempo antes de ir a impartir lecciones se aplicaban el filtro solar, el $38.3 \%$ de los encuestados respondió "inmediatamente antes", mientras que el $25.5 \%$ indicó que se aplicaba el filtro "entre 30 y 60 minutos antes" de la lección.

De las personas evaluadas, ninguna se había hecho revisar en forma periódica por un dermatólogo.

Se intentó determinar si existían antecedentes heredo familiares de cáncer de piel entre los 47 sujetos encuestados. Los datos refieren que 3 personas $(6.7 \%)$ respondieron afirmativamente, y una mencionó que el padre desarrolló dicho tipo de cáncer, sin embargo, no se obtuvieron datos de los otros dos encuestados. El tipo de cáncer reportado fue el carcinoma basocelular.

Hubo una persona con antecedentes personales patológicos por cáncer de piel. Este era de tipo no melanoma y se ubicaba en la nariz. La persona indicó que hace dos años fue tratado por carcinoma basocelular.

Al realizar el examen físico a los participantes, se encontró dermatoheliosis en 27 sujetos (34.2\%), la cual fue clasificada como leve $(22.8 \%)$ y moderada $(11.4 \%)$. Por otra parte, hubo 20 pacientes que presentaron rhitides (arrugas) (25.3\%), cloasma $(6.3 \%)$, efélides $(40.5 \%)$, hipomelanosis guttata (20.3\%), queratosis seborréicas (20.3\%), angiomas rubí $(12.7 \%)$ y telangectasias $(5.1 \%)$.
Se encontraron lesiones premalignas en 5 sujetos (6.3\%), las cuales fueron identificadas como queratosis actínicas.

Hubo casos en los que se encontraron lesiones pigmentadas. Destacan el nevus melanocítico de unión (53.2\%), intradérmico $(32.9 \%)$ y el compuesto $(8.9 \%)$. Sin embargo, también se encontraron nevus atípicos únicos $(10.1 \%)$. Un puntaje dermatoscópico de 4 fue obtenido en 7 sujetos (8.9\%). Finalmente, se reportaron 7 casos de léntigo solar (8.9\%) y dos de nevus melanocítico congénito piloso pequeño $(2.5 \%)$.

Respecto a las lesiones sugestivas de cáncer de piel, solo se encontraron dos casos (2.5\%), en los cuales se detectó una lesión sugestiva de carcinoma basocelular de tipo ulcerado $(1.3 \%)$ y una de tipo nodular $(1.3 \%)$.

Las lesiones reportadas por el médico dermatólogo fueron codificadas utilizando tres figuras. La Figura 3 resume las lesiones encontradas en sitios anatómicos sobre el cuello. Como puede observarse consistentemente, cerca del $70 \%$ de las lesiones ocurren en sitios ubicados entre las cejas y el mentón. También se reportan lesiones de entre un $6.7 \%$ y $12.1 \%$, sobre las cejas y en el cuero cabelludo. Finalmente, se refieren lesiones de entre un $6.1 \%$ y $10 \%$, entre el mentón y el cuello.

La Figura 4 permite observar el cuerpo en posición anatómica anterior y posterior. En la posición anatómica anterior sobresalen las lesiones en la región torácica superior (pecho) $(\mathrm{n}=32,40.5 \%)$, hombros $(\mathrm{n}=28,35.5 \%)$, muslos $(\mathrm{n}=23$, $29.2 \%)$, piernas $(\mathrm{n}=15,19 \%)$, brazos $(\mathrm{n}=14,17.7 \%)$ y ma$\operatorname{nos}(\mathrm{n}=9,11.4 \%)$. Por otra parte, en la posición anatómica posterior, la mayor cantidad de lesiones corresponden al tórax superior (espalda alta) y a $\operatorname{los}$ brazos $(\mathrm{n}=38,48.1 \%$ cada una). En orden descendente, siguen los hombros $(\mathrm{n}=14$, $17.9 \%)$ piernas $(\mathrm{n}=7,8.9 \%)$ manos $(\mathrm{n}=7,8.8 \%)$ y muslos $(\mathrm{n}=4,5 \%)$. No se encontraron lesiones en las plantas de los pies.

\section{Dis c us ión}

La luz solar es energía electromagnética radiante, compuesta de ondas, y radiación ultravioleta (RUV), visible e infrarroja. Dos terceras partes de la energía penetran la atmósfera y llegan a la superficie terrestre.

La RUV es el 5\% de la radiación solar terrestre que comprende ondas entre un rango de 100 a $400 \mathrm{~nm}$. La Comission Internationale de L'Eclairage (CIE) la subdivide en UVA (315-400 nm), UVB (280-315 nm) y UVC (100-280 nm); sin embargo, por conveniencia popular, la clasificación utilizada es UVC (100-290 nm), UVB (290-320 nm), UVA II (320$340 \mathrm{~nm})$ y UVA I (340-400 nm). En la superficie terrestre el 95-98\% es UVA, un 2-5\% es UVB, y la UVC es absorbida por el ozono estratosférico. La radiación UVB contribuye al $80 \%$ de los daños asociados a la exposición solar, mientras que la radiación UVA, al restante $20 \%{ }^{9,10}$. 
La exposición a la radiación UV altera parámetros inmunológicos en humanos, y el desarrollo de cáncer de piel está parcialmente bajo regulación inmunológica por ayuda de linfocitos T citotóxicos ${ }^{15}$. Las personas que pasan su tiempo a la intemperie, especialmente quienes trabajan en ambientes exteriores, normalmente presentan una mayor incidencia de carcinomas epidermoides quienes lo hacen en ambientes bajo techo, en especial asociados a exposición a radiación UVB ${ }^{16}$.

Geográficamente existe una asociación directa entre la cantidad de RUV solar y la incidencia de cáncer de piel clara ${ }^{17}$. Por su parte, el cáncer epidermoide se asocia con exposición acumulativa y constante, el de las células basales, con intermitencia y exposición durante la infancia (18). La pigmentación de la piel es un factor protector importante en cuanto a la susceptibilidad al quemado y al cáncer de piel ${ }^{19,20}$.

Es escasa la literatura que describe las lesiones de la piel en deportistas ${ }^{7,21-23}$. En Costa Rica no había sido investigada la frecuencia de lesiones cutáneas en un grupo selecto de trabajadores, los educadores físicos, por lo que se realizó el presente estudio cuando estos profesionales asistieron a su congreso profesional en marzo de 2003.

De acuerdo con la clasificación de Fitzpatrick ${ }^{8}$, el $42.6 \%$ de la muestra fue clasificada con tipos de piel I y II, lo que significa que poseen piel blanca que fácilmente tienden a quemarse, y en muy pocas ocasiones se pueden broncear.Así, casi la mitad de los encuestados presenta un riesgo importante de adquirir una lesión por exposición prolongada y crónica al sol, tal como se infiere de los datos proporcionados en el Cuadro 1, referente a las horas diarias que laboran al aire libre.

No hubo una cantidad considerable de personas con cabello rubio natural, pero sí se encontró que más del 10\% de la muestra presentaba ojos de color verde o azul. Teóricamente, estas personas tienen mayor riesgo de desarrollar cáncer de piel ${ }^{1,3,4}$.

El cáncer de piel es más frecuente en países con predominio de caucásicos y altos niveles de radiación solar ${ }^{24}$. La exposición repetida y acumulativa de la RUV en la luz solar es el principal inductor de la mayoría del cáncer no melanoma en poblaciones de piel clara ${ }^{25}$.

Como se observa en la Figura 2, los educadores físicos trabajan en horarios que abarcan los momentos cuando ocurre la mayor radiación solar del día, es decir, entre las 11:00 a.m. y las 3:00 p.m. Una sugerencia específica para las autoridades y administradores de instituciones de enseñanza, es que no se habilite el horario de 1:00 p.m. a 3:00 p.m. para impartir lecciones de educación física, ya que la RUV es mayor a esas horas.

Por otra parte, ya que en escuelas y colegios no se acatan las recomendaciones técnicas por diversas razones, al menos se podría permitir a los estudiantes y a los educadores portar prendas de vestir que les permitan reducir la RUV que absorben sus cuerpos durante las clases de educación física. Se recomienda en la literatura, que para mejorar la protección solar, particularmente durante la exposición al mediodía, o en zonas tropicales, cuando es inevitable por diferentes razones, se cubra con ropa el mayor porcentaje de la superficie corporal ${ }^{26}$. Las telas que impiden el paso de la luz visible cuando se sostienen ante el sol u otra fuente de luz, parecen ser las más protectoras contra la radiación UV ${ }^{27}$. Se ha establecido el concepto del factor de protección a la RUV (FPU), que los materiales en la fabricación de prendas determinan, como son: porosidad de la tela, tipo, color, peso y grosor (prendas hechas de más de un $70 \%$ de lana, poliéster y mezclas, especialmente si son negras, azul oscuro, blanco, verde, beige y menos de un $30 \%$ de algodón, lino y telas viscosas tienen FPU $>30^{28}$. Durante su uso existen factores que también modifican la FPU de una prenda, como son el estiramiento, la yuxtaposición a la piel y la humedad, que reducirían esta capacidad en un tercio ${ }^{29}$. Además, el hecho de emplear sustancias químicas que absorban la radiación UV al lavar las prendas, incrementa su FPU ${ }^{26}$. Se debería permitir e incentivar a que los estudiantes y profesores utilicen camisetas con mangas largas para proteger los brazos. Aunque podría ser incompatible con las políticas respecto a los uniformes de ciertas instituciones, se debería avalar el uso de gorras o sombreros, así como de anteojos oscuros para el sol y bloqueadores solares. Cambios en las políticas que permitan la utilización de sombreros y lentes para el sol, durante la exposición a la luz solar serían benéficos al reducir la exposición de piel y ojos a la RUV ${ }^{30,31}$.

Se debe evitar el uso de prendas con tejidos como mezclilla o lana y que presenten colores oscuros, ya que estos no permiten que los rayos solares se desvíen, lo que causa un mayor estrés térmico. Es preferible el uso de prendas de algodón (gruesas) y de color claro (p. ej: blanco, gris), las cuales presentan un coeficiente de reflexión mayor que las oscuras (p. ej: negras) ${ }^{32,33}$.

El uso de filtros solares disminuye la RUV absorbida por la piel al reflejar por reflexión o absorción y luego reemisión como calor ${ }^{34}$. Los humanos expuestos a la RUV, utilizando cantidades suficientes de filtro solar, no se queman ${ }^{35}$. Se ha encontrado que el uso frecuente y correcto de filtros solares en humanos, disminuye significativamente la incidencia de queratosis actínicas (lesiones premalignas) ${ }^{36}$. En este estudio se determinó que los educadores físicos no conocen la diferencia existente entre una loción bronceadora y un filtro solar, lo que refuerza los hallazgos en la literatura mundial, de que los pacientes que "utilizan" filtros solares presentan más cáncer de piel que los que no lo hacen ${ }^{37}$, pero esto se ha justificado por observaciones en cuanto a que no lo aplican antes de la exposición ${ }^{38}$, temprano antes de la exposición ${ }^{39}$, o no utilizan una capa adecuadamente gruesa, lo que disminuye la protección a la mitad ${ }^{40}$.Aún más, un porcentaje muy bajo de las personas encuestadas manifestó utilizar lociones bronceadoras o filtros solares pocas veces durante el día, lo 
que indica que se debe promover más el uso de estos filtros solares, así como de cremas para el cuidado regular de la piel. Es necesaria la asesoría de un dermatólogo que recomiende un producto específico que satisfaga las necesidades de cada persona (FPU adecuado, costo/beneficio). También, se debe realizar una campaña para promover el uso continuo de los filtros solares, ya que se observó que la mayoría de las personas se aplicaban los productos inmediatamente antes de ir a impartir las lecciones, por lo que se requiere reforzar las instrucciones que el fabricante o el médico indiquen para un uso apropiado y eficaz del producto, conociendo que la dosis de RUV inmunosupresora es menor que la dosis requerida para inducir una quemadura ${ }^{41}$.

La educación en cuanto al uso también aporta beneficios, no solo a nivel de medicina de prevención secundaria o primaria, sino también a nivel estético para el paciente, ya que se ha demostrado que la utilización de filtros solares puede proveer una protección equivalente a una reducción de 40 años en exposición no protegida, durante una vida de 70 años ${ }^{42}$.

Un hallazgo de este estudio fue que 3 personas indicaron tener antecedentes familiares de cáncer de piel, factor de riesgo relevante para el desarrollo de este. Respecto a los antecedentes personales patológicos de cáncer de piel, se encontró que una persona presentaba un carcinoma basocelular de tipo no melanoma, el cual es el más común de los tipos de cáncer. Por otra parte, las lesiones dermatológicas mencionadas están asociadas a la exposición solar, no así directamente fotoinducidas; adicionalmente, en algunas de ellas, su existencia y una exposición solar prolongada y crónica, representan un riesgo para el desarrollo de cáncer de piel, por lo que se creyó pertinente reportarlas en este estudio.

En deportistas profesionales, como los triatlonistas, quienes forman una población que se expone al sol aproximadamente $20 \mathrm{~h} \cdot \mathrm{sem}^{-1}$, se ha encontrado que durante las competencias, las zonas de los muslos y hombros son las que sufren mayores quemaduras solares ${ }^{43}$. En este estudio, y como se desprende del Cuadro 1, se determinó que, en promedio, los educadores físicos se exponen al sol cerca $25 \mathrm{~h} \cdot \mathrm{sem}^{-1}$, y la mayoría de lesiones cutáneas se localizaron en la zona torácica superior, específicamente en el pecho y la espalda alta, aunque también en los hombros y los muslos se reportaron porcentajes importantes. Sin embargo, el mayor porcentaje ocurrió en la zona comprendida entre las cejas y el mentón. Este hallazgo indica que los educadores físicos deben poner especial atención al cuidado de su rostro.

En resumen, el estudio transversal pretendió describir los hallazgos cutáneos más frecuentes en los educadores físicos. Se recomienda realizar investigaciones que evalúen objetivamente la cantidad de RUV que en general, y en distintos meses, reciben los educadores físicos y sus estudiantes, durante las lecciones y en otras actividades realizadas al aire libre, como por ejemplo, festivales, ferias, y competencias. Se sugiere tomar medidas para la prevención del cáncer de piel, como por ejemplo, el uso de prendas de vestir protectoras, lociones bloqueadoras, anteojos oscuros, sombreros o gorras, y "negociar" el horario de trabajo para evitar las horas de mayor radiación solar.

\section{Abstract}

Purpose: In Costa Rica, physical education professionals are chronically exposed to solar radiation throughout their lives. However, skin lesions in this group of workers have not been described so far.The purpose of this study was to describe the incidence of skin lesions in this professionals in Costa Rica.

Methods: Twenty-three males (48.9\%) and twenty-four females $(51.1 \%)$ volunteers answered a questionnaire and were examined by a dermatologist from the Dermatology Service of the Rafael Angel Calderon Guardia Hospital. Information on demographics, phototype, general health status, work schedule, clothing, skin and sun protection, family and personal history for skin cancer, and anatomic region of the skin lesion were obtained.

Results and discussion: In general, most lesions were found in the face, chest, shoulders, back, arms, and thighs. There were 3 cases of family history of skin cancer; and one case of personal history (non melanoma) of skin cancer. Pre-malignant lesions suggestive of skin cancer were found in 5 cases $(6.3 \%)$. In conclusion, avoiding excessive sun exposure and a yearly visit to a dermatologist is recommended. It is also important to perform a monthly self-examination of the skin, to wear sun glasses, hat, sun blockers, and thick-layered light-colored preferably cotton clothes.

\section{Agradecimientos}

Un agradecimiento especial a los educadores físicos que voluntariamente participaron en este estudio; al comité organizador del Congreso Nacional de Educación Física, por facilitarnos una sala adecuada para la revisión médica; al Dr. Daniel Rodríguez Guerrero, Jefe del Servicio de Medicina del Hospital Rafael Ángel Calderón Guardia (HRACG); al Dr. Luis Paulino Hernández Castañeda, Director del HRACG; y al Dr. Alvaro Chen Chen, jefe del Servicio de Dermatología del HRACG., por brindar los permisos correspondientes para que los médicos participaran en el estudio. 


\section{Referencias}

1. Tortora GJ, Grabowski SR. Principios de anatomía y fisiología (2da reimpresión). España: Harcourt Brace de España, S.A., 1993.

2. Jerant AF, Johnson JT, Sheridan CD, Caffrey TJ. Early detection and treatment of skin cancer. American Family Physician 2000, 62: 357368, 375-376, 381-382.

3. Rosso S, Joris F, Zanetti R. Risk of basal and squamous cell carcinomas of the skin in Sion, Switzerland: a case-control study.Tumori 1999; 85: 435-442.

4. van Dam RM, Huang Z, Rimm EB, Weinstock MA, Spiegelman D, Colditz GA, Willett WC, Giovannucci E. Risk factors for basal cell carcinoma of the skin in men: results from the health professionals followup study.American Journal of Epidemiology 1999; 150: 459-468.

5. Bartels R, Vargas RM, Muñoz G. Incidencia y mortalidad del cáncer en Costa Rica: 1994 - 1996. San José, Costa Rica: Ministerio de Salud; 1999, pp. 1-143.

6. Muglia JJ, Pesce K, McDonald CJ. Skin cancer screening: A growing need. Surg Oncol Clin N Am 1999; 8: 735-745.

7. Moncada-Jiménez, J. La radiación ultravioleta y la piel del deportista. Revista Educación 2003; 27(2), en prensa.

8. Fitzpatrick TB. The validity and practicality of sun reactive skin type I through VI. Arch Dermatol 1988; 124: 869.

9. McGregor JM, Hawk JLM. Acute effects of ultraviolet radiation on the skin. En Irwing Freedberg et al. (Eds), Dermatology in General Medicine 5th Ed. New York, NY: McGraw-Hill; 1999, pp. 1562-1568.

10. Diffey BL. What is Light? Photodermatol Photoinmunol Photomed 2002; 18: 68-74.

11. Albert MR. Dermatology related advertisements form 1920s to 1930s. Int J Dermatol 2000; 39: 389-395.

12. You YH, Szabó PE, Pfeifer GP. Cyclobutane pyrimidine dimmers form preferentially at the major p53 mutational hotspot in UVB-induced mouse skin tumors. Carcinogenesis 2000; 21: 2113-2117.

13. Urbach F. Ultraviolet carcinogenesis: Experimental, global and genetic aspects in Sunlight and Man: Normal and Abnormal Photobiologic responses. En Pathak MAet al. (Eds.). Tokyo: University of Tokyo Press; 1974, pp. 259.

14. The National Academy of Sciences. Protection against depletion of stratospheric ozone by chlorofluorocarbons (Appendix F). Washington, DC: The National Academy of Sciences; 1979, pp. 325.

15. Granskin RD. Photoinmunology in dermatology. En Irwing Freedberg et al. (Eds.), Dermatology in General Medicine 5th Ed. New York, NY: McGraw-Hill; 1999, pp. 1562-1568.

16. Vitasa BC, Taylor HR, Strickland PT, Rosenthal FS, West S, et al. Association of non melanoma skin cancer and actinic keratosis with cumulative solar ultraviolet exposure in Maryland watermen. Cancer 1990; 65: 2811

17. Abarca JF, Casiccia CC. Skin cancer and ultraviolet-B radiation under the Antartic ozone hole: southern Chile, 1987-2000. Photodermatol Photoimmunol Photomed 2002; 18: 294-302.

18. Kricker A, Armstrong BK, English DR, Heenan PJ. Does intermittent sun exposure cause basal cell carcinoma? Acase control study in Western Australia. Int J Cancer 1995; 60: 489.

19. Kricker A, Armstrong BK, English DR. Sun exposure and non-melanocytic skin cancer. Cancer Cases Control 1994; 5: 367.

20. English JS, Swerdlow AJ. The risk of malignant melanoma, internal malignancy and mortality in xeroderma pigmentosum patients. Br J Dermatol 1987; 117: 457-461.
21. Ballestero A. Patología cutánea en la actividad física y deportiva. Piel 1988; 3: 229-238.

22. Erickson JG, von Gremmingen GR. Surfer's nodules and other complications of surf-boarding. JAMA1967; 201: 134-136.

23. Moehrle M, Heinrich L, Schmid A, Garbe C. Extreme UV exposure of professional cyclists. Dermatology 2000; 201: 44-45.

24. Kripke MC. Carcinogenesis: Ultraviolet Radiation. En Irwing Freedberg et al. (Eds.), Dermatology in General Medicine 5th Ed. New York, NY: McGraw-Hill; 1999, pp. 465-460.

25. Fears TR, Scotto J, Schneiderman MA. Mathematical models of age on ultraviolet effects on the incidence of skin cancer among whites in the USA. Am J Epidemiol 1977; 105: 420.

26. Gambichler T,Altmeyer P, Hoffman K. Role of clothes in sun protection. Recent Results Cancer Res 2002; 160: 15-25.

27. Davis S, Capjack L, Kerr N. et al. Clothing as protection from ultraviolet radiation: which fabric is most effective. Int J Dermatol 1997; 36: 374-379.

28. Gambichler T, Rotterdam S, Altmeyer P, Hoffman K. Protection against ultraviolet radiation by commercial summer clothing: need for standardized testing and labeling. BMC Dermatol 2001; 1: 6 .

29. Jevtic AP. The sun protective effect of clothing, including beachwear. Australian J Dermatol 1990; 31: 5-7.

30. Rosenthal FS, Phoon C, Bakalian AE, Taylor HR. The ocular dose of ultraviolet radiation to outdoor workers. Invest Ophthalmol Vis Sci 1988; 29: 649-656

31. Wong JCF,Airey DK, Fleming RA. Annual reduction of solar UV exposure to the facial area of outdoor workers in southeast Queensland by wearing a hat. Photodermatol Photoinmunol Photomed 1997; 13: 186188.

32. Moehrle M, Garbe C. Solar UV-protective properties of textiles. Dermatology 2000; 201: 82 .

33. Pandolf KB, Sawka MN, Gonzalez RR. Human performance physiology and environmental medicine at terrestrial extremes. Carmel, IN: Cooper Publishing Group; 1986.

34. Shaath NA. On the theory of ultraviolet absorption by sunscreen chemicals. J Soc Cosmet Chem 1987; 82: 193-198.

35. Kaldbey KH. The photoprotective potential of the new superpotent suncreens. J Am Acad Dermatol 1990; 22: 449-452.

36. Thompson SC, Jolley D, Marks R. Reduction of solar keratoses by regular sunscreen use. N Engl J Med 1993; 329: 1147-1151.

37. Autier P, Doré JF, Schifflers E et al. Melanoma and use of sunscreens: an EORTC case-control study in Germany. Int J Cancer 1995; 61: 749755 .

38. Robinson JK, Rademaker AW. Sun protection by families at the beach. Arch Pediatr Adolesc Med 1998; 152: 466-470.

39. Diffey BL. When should sunscreen be reapplied? J Am Acad Dermatol 2001; 45: 882-885

40. Bech-Thomsen N, Wulf HC. Sunbathers' application of sunscreen is probably inadequate to obtain the sun protection factor assigned to the preparation. Photodermatol Photoimmunol Photomed 1992; 9: 242244

41. Kelly DA, Young AR, McGregor JM, Seed PT, Potten CS, Walker SL. Sensitivity to sunburn is associated with susceptibility to ultraviolet radiation-induced suppression of cutaneous cell-mediated immunity. J Exp Med 2000; 191: 561-566.

42. Diffey BL. Is daily use of sunscreens of benefit in the UK? Br J Dermatol 2002; 146: 659-662.

43. Moehrle M. Ultraviolet exposure in the Ironman triathlon. Med and Sci Sports Exerc 2001; 33:1385-1386. 